\title{
Pulsating Degenerate Stars in the EC Survey
}

\author{
R. S. Stobie, C. Koen, D. Kilkenny
}

South African Astronomical Observatory, PO Box 9, Observatory 7935, Cape, South Africa

D. O'Donoghue

Department of Astronomy, University of Cape Town, Rondebosch 7700, Cape, South Africa

\section{Introduction}

For asteroseismological reasons the discovery of new pulsating degenerate stars is important. Bradley (1993) lists the number of known pulsating white dwarfs at that time - 23 known DAV (ZZ Ceti) stars, 7 known DBV stars and 5 known DOV (PG1159) stars. Of these degenerate pulsators $80 \%$ (including all DBVs) are in the northern hemisphere. This illustrates the great incompleteness in the search for such objects in the southern hemisphere.

The Edinburgh-Cape Blue Object Survey is a potential source of candidate degenerate pulsators in the southern hemisphere (Stobie et al. 1992). The blue stellar objects are identified from COSMOS scans of U and B plates taken with the UK Schmidt Telescope. Follow-up photometry and spectroscopy are being obtained at the South African Astronomical Observatory (SAAO) to classify and determine the nature of each blue stellar object. Currently, over 190 DA white dwarfs have been identified, of which the majority are not in existing catalogues (O'Donoghue et al. 1993). From these white dwarfs, we have selected DA stars with B-V colours near or within the range $0.15 \leq \mathrm{B}-\mathrm{V} \leq 0.25$ together with all the non-DA degenerate stars (DO, DB, etc.) to monitor for variability.

\section{New pulsating degenerate stars}

High speed photometry with 10 s time resolution in white light has been obtained with the SAAO $1.0-\mathrm{m}$ and $0.75-\mathrm{m}$ telescopes at Sutherland. To detect variability at least 1.5 hours high speed photometry is obtained on two different nights and the time series photometry is frequency analysed. So far we have examined for pulsation $39 \mathrm{DA}$ white dwarfs close to the instability strip with 4 new variables detected (3 DAs and $1 \mathrm{DAZ}$ ) and $16 \mathrm{DO} / \mathrm{DB}$ degenerates with 1 new $\mathrm{DB}$ variable detected. The results will be presented only for $2 \mathrm{DAVs}$ and $1 \mathrm{DBV}$ as the other stars require further observation and analysis.

EC23487-2424 and EC14012-1446 are two large-amplitude DAV pulsators. The data and analysis of EC23487-2424 have already been described (Stobie et al. 1993). The light curve is non-sinusoidal, with a peak-to-peak variation ranging from 0.06 to $0.24 \mathrm{mag}$ in white light. Six nights of observation show a multiple-period structure, with periods between 800 and 1000 s. The principal 
frequency at $f=1.101 \mathrm{mHz}$ is present on most nights observed with variable amplitude, probably caused by the presence of multiple close frequencies.

Photometry of EC14012-1446 shows the non-sinusoidal nature of the light curve with a peak-to-peak variation ranging from 0.08 to 0.30 mag in white light. The periodogram from 0 to $10 \mathrm{mHz}$ of the first night of photometry exhibits at least 8 frequencies well above the noise. It is apparent that some of these frequencies are either harmonics or combination terms (e.g., $2 f_{1}, f_{1}+f_{2}$, $f_{1}+f_{4}$ ) resulting in the identification of five independent frequencies at 1.067 , $1.382,1.639,1.885$ and $2.507 \mathrm{mHz}$. The frequency analyses of all four nights shows that these frequencies are present on all nights, although there is strong evidence that the amplitude of the dominant frequency (at least) is variable.

The asteroseismological understanding of individual DAV stars has long resisted interpretation for two reasons (a) the low-amplitude pulsators do not reveal sufficient modes to enable analysis (b) the large-amplitude pulsators have power spectra which change radically on many timescales. Recently, however, Clemens (1993) has shown that progress can be made by considering the DAV stars as a group rather than as individuals. He showed that the hottest DAVs have the shortest periods and smallest amplitudes. Both period and amplitude increase monotonically with decreasing temperature, consistent with theory.

A remarkable result found by Clemens (1993) was that the periods of the DAVs as a group form a highly non-random distribution in period space with strong clusterings near preferred values. EC14012-1446 (and to some extent EC23487-2424) fits this pattern. These results are interpreted as evidence that the DAV stars are remarkably homogeneous as a group with similar total mass and similar hydrogen layer mass $\left(\mathrm{M} \sim 10^{-4} \mathrm{M}_{*}\right)$. As all DA stars pass through the instability strip at some stage in their evolution this conclusion holds for all DA stars.

EC20058-5234 is the eighth pulsating helium white dwarf to be discovered. More than 10 periodicities have been discovered in about 52 hours of high speed photometry, the most prominent being at 281 and 257s. Many of the periodicities appear to be permanent features of the light curve, but the amplitudes vary: the strongest mode has been observed at amplitudes between 6 and $12 \mathrm{mmag}$. EC20058-5234 is exceptional in the group of DBVs both because of its short period and its low amplitude.

\section{References}

Bradley, P. A. 1993, in Proc. 2nd WET Workshop, E. Meistas \& J. Solheim, Baltic Astronomy, 2, 559

Clemens, J. C. 1993, in Proc. 2nd WET Workshop, E. Meistas \& J. Solheim, Baltic Astronomy, 2, 407

O'Donoghue, D., Chen, A., Kilkenny, D., \& Stobie, R. S. 1993, in Proc. 8th European Workshop on White Dwarfs, M. Barstow, Kluwer, 39

Stobie, R. S., Chen, A., Kilkenny, D., \& O'Donoghue, D. 1992, in Variable Stars and Galaxies, B. Warner, ASP Conf. Ser., 30, 87

Stobie, R. S., Chen, A., O'Donoghue, D., \& Kilkenny, D. 1993, MNRAS, 263, L13 